\title{
The Impact of CYP24A1 Polymorphisms on Hypertension Susceptibility
}

\author{
Qiuhong Bao Dan Wang Yong Zhang Lili Bao Haiyu Jia
}

Gerontological Center, Affiliated Hospital of Inner Mongolia Medical University, Hohhot, China

\author{
Keywords \\ CYP24A1 · Hypertension · Case-control study · Genetic variations · Risk
}

\begin{abstract}
Background: Hypertension is one of the leading causes of human death and disability. CYP24A1 regulates vitamin D activity and is closely linked to hypertension. However, the relationship between CYP24A1 polymorphisms and hypertension risk remains unclear. Methods: This case-control study included 503 hypertensive patients and 498 healthy controls from the Chinese Han population. The genotypes of CYP24A1 polymorphisms were detected using the Agena MassARRAY method. The association between genetic variations of CYP24A1 and hypertension risk was evaluated with odds ratios (OR) and $95 \%$ confidence intervals (CI) in genetic models. Results: We found that rs 56229249 of CYP24A1 significantly decreased the hypertension risk in homozygote (OR $0.51,95 \% \mathrm{Cl} 0.29-0.91, p=0.022)$ and recessive models (OR $0.51,95 \% \mathrm{Cl} 0.29-0.91, p=0.023$ ). Further stratification analyses indicated that hypertension risk is related to age and sex, rs2762934 polymorphism increases hypertension risk among younger subjects (<61 years), and rs1977297 influences the risk of hypertension among older subjects ( $\geq 61$ years). In addition, rs 2762940 is related to hypertension risk in men, and rs56229249 is a protective factor against hypertension in women. Conclusions: Our study suggests that genetic variations of the CYP24A1 gene were significantly associated with susceptibility to hypertension in the Chinese population.

(c) 2020 The Author(s)

Published by S. Karger AG, Basel
\end{abstract}

\section{Introduction}

Hypertension is the most common disease that affects human beings worldwide, accounting for 2.5 million deaths $(27.5 \%$ of total deaths) in 2013 in China [1, 2]. Compared with healthy subjects, individuals with a high blood pressure (BP) stand a higher risk of some 


\section{Kidney \\ Blood Pressure \\ Research}

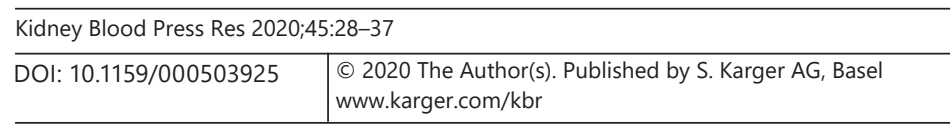

Bao et al.: The Impact of CYP24A1 Polymorphisms on Hypertension Susceptibility

diseases such as stroke, coronary heart disease (CHD), heart failure, and kidney disease [3, 4]. The complex mechanisms of BP regulation are determined by tight interactions between various genetic and environmental factors [5, 6]. Approximately $30-70 \%$ variability in human hypertension is attributed to multiple genetic factors [7].

CYP24A1 (cytochrome P450 family 27 subfamily A member 1 ) is a gene located on chromosome 20 (q13.2) encoding the primary catabolic enzyme for circulating 1,25-dihydroxyvitamin D and 25-hydroxyvitamin D [8]. CYP24A1 is involved in regulating the level of vitamin $D$. The role of vitamin $D$ in the etiology of hypertension and cardiovascular disease has been increasingly identified $[9,10]$. Previous studies indicated that vitamin D deficiency can lead to hypertension [11]. CYP24A1, as a gene in the vitamin D pathway, showed significant association with systolic BP, diastolic BP, or mean arterial pressure [12]. CYP24A1 polymorphisms may modify the effect of vitamin D on BP. It has revealed that inactivating mutations of the CYP24A1 gene in individuals are associated with the level of 1,25-dihydroxyvitamin D and parathyroid hormone (PTH) [13], hypercalcemia, hypercalciuria [14], kidney disease [15], but little research has been conducted on CYP24A1 polymorphisms for influencing hypertension risk in the Chinese Han population. To further address the role of CYP24A1 in hypertension development, we conducted a case-control study to investigate whether genetic variants in CYP24A1 are related to hypertension risk.

\section{Methods}

\section{Study Participants}

The study consisted of 503 hypertensive subjects (303 males and 200 females) and 498 healthy controls with normotension (298 males and 200 females), who were recruited from the Affiliated Hospital of Inner Mongolia Medical University, Hohhot, China. Hypertension was defined as a seated systolic and/or diastolic BP >140 and/or $90 \mathrm{~mm} \mathrm{Hg}$ in at least two separate measurements by professional physicians. All hypertension patients had primary hypertension. Patients who had hypertension treatment with medications, secondary hypertension, pregnancy, inflammation, or other autoimmune diseases were excluded from this study. The control group consisted of subjects without hypertension, diabetes mellitus, tumors, inflammation-related diseases, cardiovascular diseases, or other obvious diseases. The clinical characteristics, including urea, creatinine, uric acid (UA), and lipid profile, were collected from their medical records.

\section{Selection of Single Nucleotide Polymorphisms and Genetic Analysis}

The selection of human CYP24A1 gene single nucleotide polymorphisms (SNPs; rs2762934, rs1977297, rs2762940, rs4809958, rs56229249, and rs2585428) was based on previous studies $[16,17]$ and the data from the 1000 Genomes Project. Blood samples were collected from all participants and were stored in EDTA-containing vacutainers. Genomic DNA was extracted from whole blood using the GoldMag Mini Purification Kit (GoldMag Co. Ltd., Xi'an, China). Genotyping was performed using the MassARRAY iPLEX Gold Assay (Agena Bioscience, San Diego, CA, USA) [18]. The primer sequences used for genotyping the CYP24A1 polymorphisms are shown in online supplementary Table 1 (for all online suppl. material, see www.karger.com/doi/10.1159/000503925).

\section{Statistical and Bioinformatics Data Analyses}

Statistical analysis was performed using SPSS 18.0 for Windows (SPSS, Chicago, IL, USA). Continuous variables are expressed as the mean \pm standard deviations (SD) and a $t$ test was performed to compare the differences in these variables between all participants. Categorical 


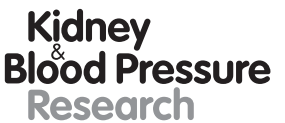

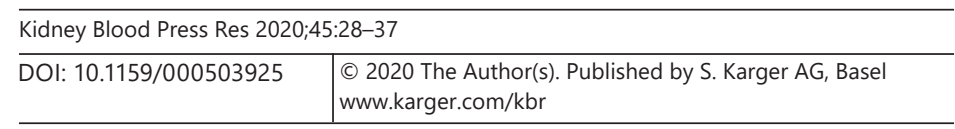

Bao et al.: The Impact of CYP24A1 Polymorphisms on Hypertension Susceptibility

variables were assessed by $\chi^{2}$ test. In the control group, genotype distributions for each SNP were analyzed using the $\chi^{2}$ test to see if they deviated from Hardy-Weinberg equilibrium. We assessed the association between CYP24A1 polymorphisms and hypertension by odds ratios (OR) with 95\% confidence intervals (CI) using logistic regression analysis [19]. Multiple models were analyzed by PLINK software, including homozygote, heterozygote, dominant, recessive, and additive models [18]. Haplotype analysis and linkage disequilibrium (LD) were evaluated by the PLINK software. Besides that, we used HaploReg v.4.1 (https://pubs. broadinstitute.org/mammals/haploreg/haploreg.phpto) to predict the possible functions on these CYP24A1 SNPs. All of the reported $p$ values are based on 2-sided tests, and $p<0.05$ was considered to be statistically significant.

\section{Results}

\section{Basic Characteristics of the Subjects}

The characteristics for all study subjects are summarized in Table 1. The mean ages of hypertensive cases and healthy controls were $63.05 \pm 10.81$ and $60.69 \pm 6.38$ years, respectively. No significant difference was observed between the two groups in regard to sex, urea, triglyceride, total cholesterol, and high-density lipoprotein cholesterol. However, the values of creatinine, UA, and low-density lipoprotein cholesterol in the patient group were significantly different compared with those in the control group. The smoking and drinking status of the two groups are presented in Table 1. Among the patient group, 291 individuals (58\%) had CHD and 202 individuals (40\%) had cerebral infarction.

\section{Genotyping and Candidate SNP Details}

Six SNPs in the CYP24A1 gene (rs2762934, rs1977297, rs2762940, rs4809958, rs56229249, and rs2585428) were successfully genotyped. The details of these candidate SNPs are shown in Table 2. All studied variants complied with the Hardy-Weinberg equilibrium $(p>0.05)$. Moreover, each selected SNP had more than $5 \%$ minor allele frequency in the Chinese population. However, there were no strong relationships between CYP24A1 polymorphisms and hypertension risk in the allele model $(p>0.05)$. We also found that candidate SNPs were related to the regulation of enhancer histone marks, DNAse, proteins bound, and motifs changed by HaploReg (version 4.1).

\section{Association of the Candidate SNPs with Hypertension}

As shown in Table 3, homozygous rs56229249 variants had a significantly decreased risk of hypertension (OR $0.51,95 \%$ CI $0.29-0.91, p=0.022$ ) compared to subjects with homozygous wild-type alleles. The SNP rs56229249 was also associated with a significantly lower risk of hypertension in a recessive model (OR 0.52, 95\% CI 0.29-0.91, $p=0.023$ ). However, no significant association was detected between the remaining 5 SNPs and hypertension risk in the Chinese population $(p>0.05)$.

\section{Association of the Candidate SNPs with Hypertension in Stratified Subgroups}

In Table 4, the genotype AG of SNP rs2762934 is associated with an increased risk of hypertension in the younger subgroup (age <61 years; OR 1.96, 95\% CI 1.18-3.27, $p=0.010$ ). For subjects aged 61 years or older, rs1977297 was associated with a higher risk of hypertension in multiple models (allele: OR 1.35, 95\% CI 1.05-1.73, $p=0.018$; dominant: OR 1.44, $95 \%$ CI 1.01-2.06, $p=0.046$; additive: OR 1.35, 95\% CI 1.02-1.78, $p=0.034$ ). Moreover, a significant difference in the distribution of genotype AC was identified between the hypertensive and healthy men (OR 1.42, 95\% CI 1.01-2.00, $p=0.041$ ). Women who had 
Kidney

Blood Pressure

Research

Table 1. Characteristics of hypertension patients and healthy controls

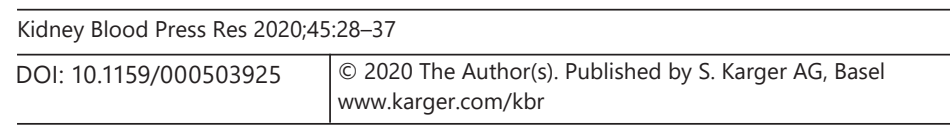

Bao et al.: The Impact of CYP24A1 Polymorphisms on Hypertension Susceptibility

\begin{tabular}{|c|c|c|c|}
\hline & $\begin{array}{l}\text { Hypertensive cases } \\
(n=503)\end{array}$ & $\begin{array}{l}\text { Healthy controls } \\
(n=498)\end{array}$ & $p$ \\
\hline Age & $63.05 \pm 10.81$ & $60.69 \pm 6.38$ & $<0.001$ \\
\hline$\geq 61$ years & $300(60)$ & 282 (57) & \\
\hline$<61$ years & $203(40)$ & $216(43)$ & \\
\hline Sex & & & 0.949 \\
\hline Male & $303(60)$ & $298(60)$ & \\
\hline Female & $200(40)$ & $200(40)$ & \\
\hline Urea & $5.50 \pm 2.26$ & $7.03 \pm 21.29$ & 0.110 \\
\hline Creatinine & $77.26 \pm 24.62$ & $68.06 \pm 34.36$ & $<0.001$ \\
\hline Uric acid, $\mu \mathrm{mol} / \mathrm{L}$ & $289.04 \pm 97.88$ & $327.78 \pm 81.56$ & $<0.001$ \\
\hline $\mathrm{TG}, \mathrm{mmol} / \mathrm{L}$ & $1.68 \pm 1.29$ & $1.78 \pm 1.21$ & 0.284 \\
\hline $\mathrm{TC}, \mathrm{mmol} / \mathrm{L}$ & $18.53 \pm 327.32$ & $4.85 \pm 5.48$ & 0.466 \\
\hline HDL, mmol/L & $1.10 \pm 0.24$ & $1.09 \pm 0.31$ & 0.588 \\
\hline LDL, mmol/L & $2.56 \pm 0.72$ & $1.86 \pm 0.83$ & $<0.001$ \\
\hline \multicolumn{4}{|l|}{ Smoking status } \\
\hline Yes & $105(21)$ & $103(21)$ & \\
\hline No & $45(9)$ & $186(37)$ & \\
\hline Absent & $343(70)$ & $209(42)$ & \\
\hline \multicolumn{4}{|l|}{ Drinking status } \\
\hline Yes & $66(13)$ & 97 (19\%) & \\
\hline No & $92(18)$ & $180(36$ & \\
\hline Absent & 345 (69) & $221(45)$ & \\
\hline \multicolumn{4}{|l|}{ CHD } \\
\hline Yes & $291(58)$ & & \\
\hline No & $212(42)$ & & \\
\hline \multicolumn{4}{|l|}{ Cerebral infarction } \\
\hline Yes & $202(40)$ & & \\
\hline No & $301(60)$ & & \\
\hline
\end{tabular}

Data are presented as the mean \pm SD or $n(\%)$. Bold $p$ values are significant. HDL, high-density lipoprotein; LDL, low-density lipoprotein; CHD, coronary heart disease.

rs56229249-G were less likely to suffer from hypertension (OR 0.60, 95\% CI 0.43-0.83, $p=$ 0.002). In the female subgroup, there was also a strong link between rs56229249 and hypertension susceptibility in genetic models (codominant: OR $0.23,95 \%$ CI $0.09-0.59, p=0.003$; dominant: OR $0.60,95 \%$ CI $0.40-0.90, p=0.015$; recessive: OR $0.26,95 \%$ CI $0.10-0.67, p=$ 0.005 ; additive: OR $0.59,95 \% \mathrm{CI} 0.42-0.82, p=0.002$ ). No notable relationships of rs 4809958 and rs2585428 with hypertension risk were identified in the stratified subgroups, so we have not provided these data. We subsequently performed an analysis on CYP24A1 polymorphisms and hypertension in the cardiovascular disease subgroups. As shown in online supplementary Table 2, rs56229249 of CYP24A1 might be associated with coexisting hypertension and CHD.

\section{Haplotypes and LD pattern}

We further conducted the LD and haplotypes analyses on the CYP24A1 polymorphisms. It revealed two blocks (block 1: rs2762934 and rs1977297; block 2: rs2762940 and rs4809958) in CYP24A1 (Fig. 1). The association between the haplotype of CYP24A1 and hypertension susceptibility is presented in Table 5. No significant association was found.

\section{CYP24A1 Polymorphisms and Clinical Factors}

In online supplementary Table 3 , we analyzed the association between genotypes of CYP24A1 polymorphisms and clinical factors. Hypertension patients with different genotypes 
Kidney

Blood Pressure

Research
Kidney Blood Press Res 2020;45:28-37 DOI: $10.1159 / 000503925$

(c) 2020 The Author(s). Published by S. Karger AG, Base www.karger.com/kbr

Bao et al.: The Impact of CYP24A1 Polymorphisms on Hypertension Susceptibility
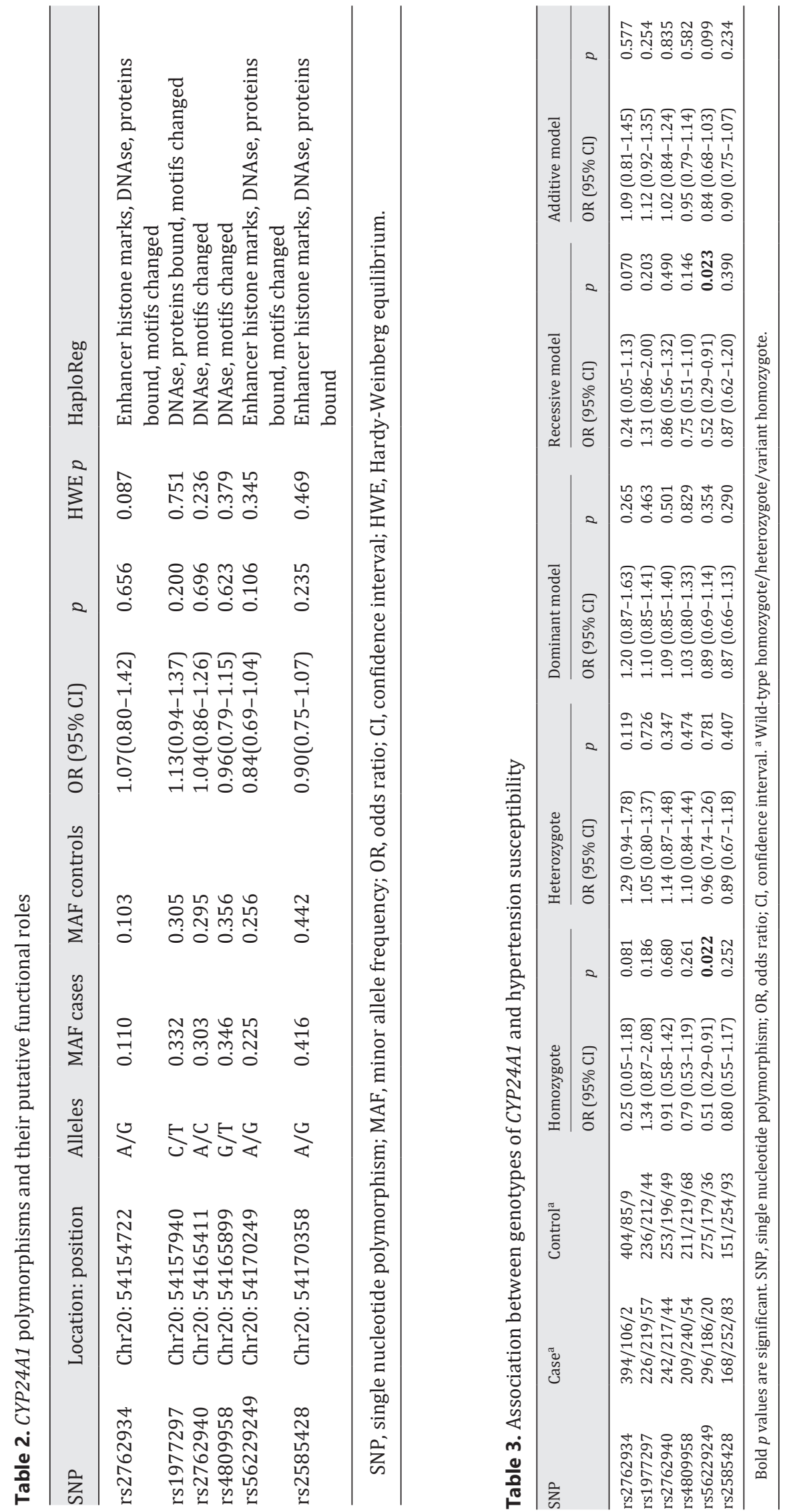


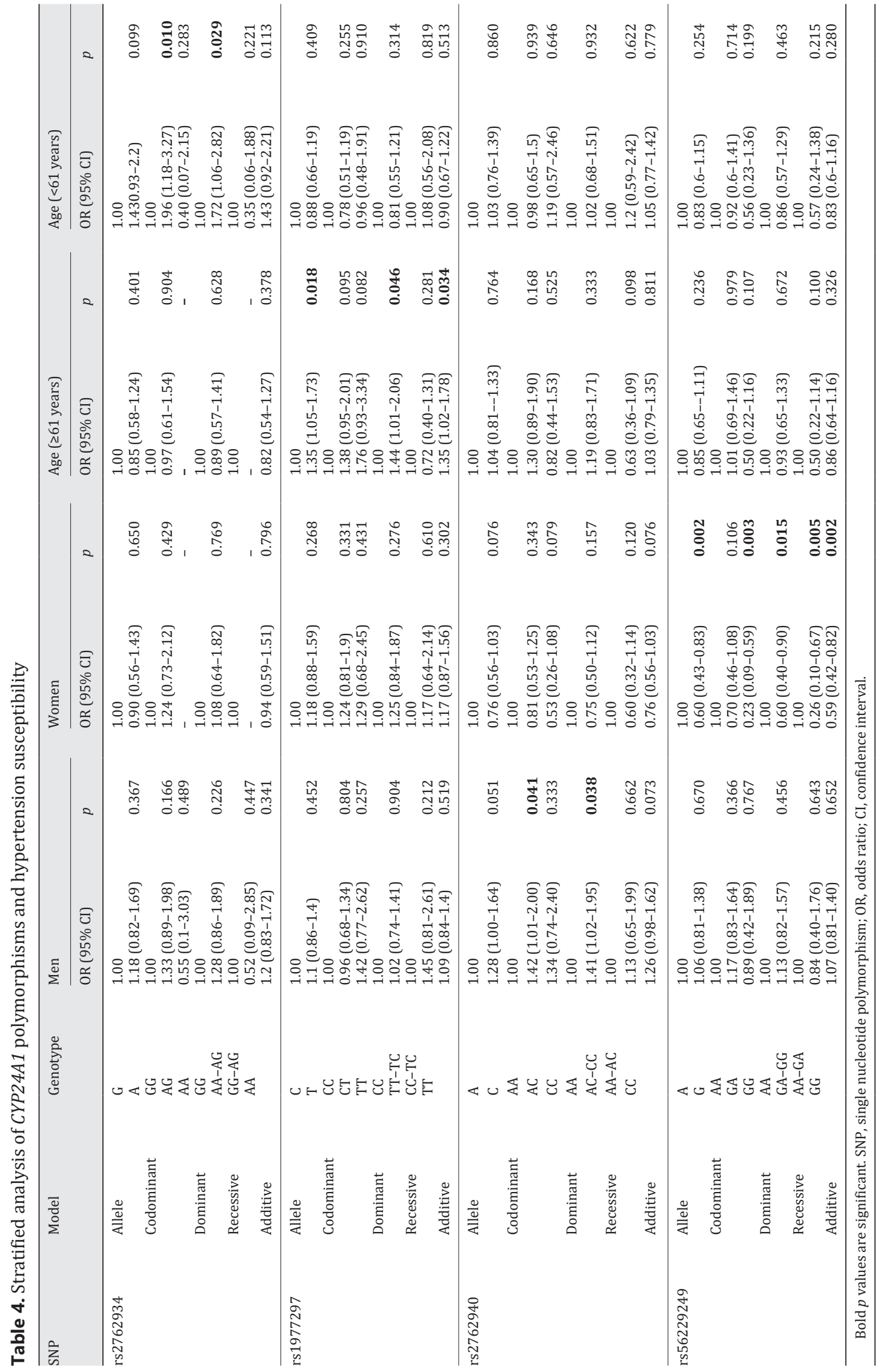


Fig. 1. Haplotype block map for the genetic variations of CYP24A1. Block 1 includes rs2762934 and rs1977297, block 2 includes rs2762940 and rs4809958. The LD between 2 SNPs is standardized D'.

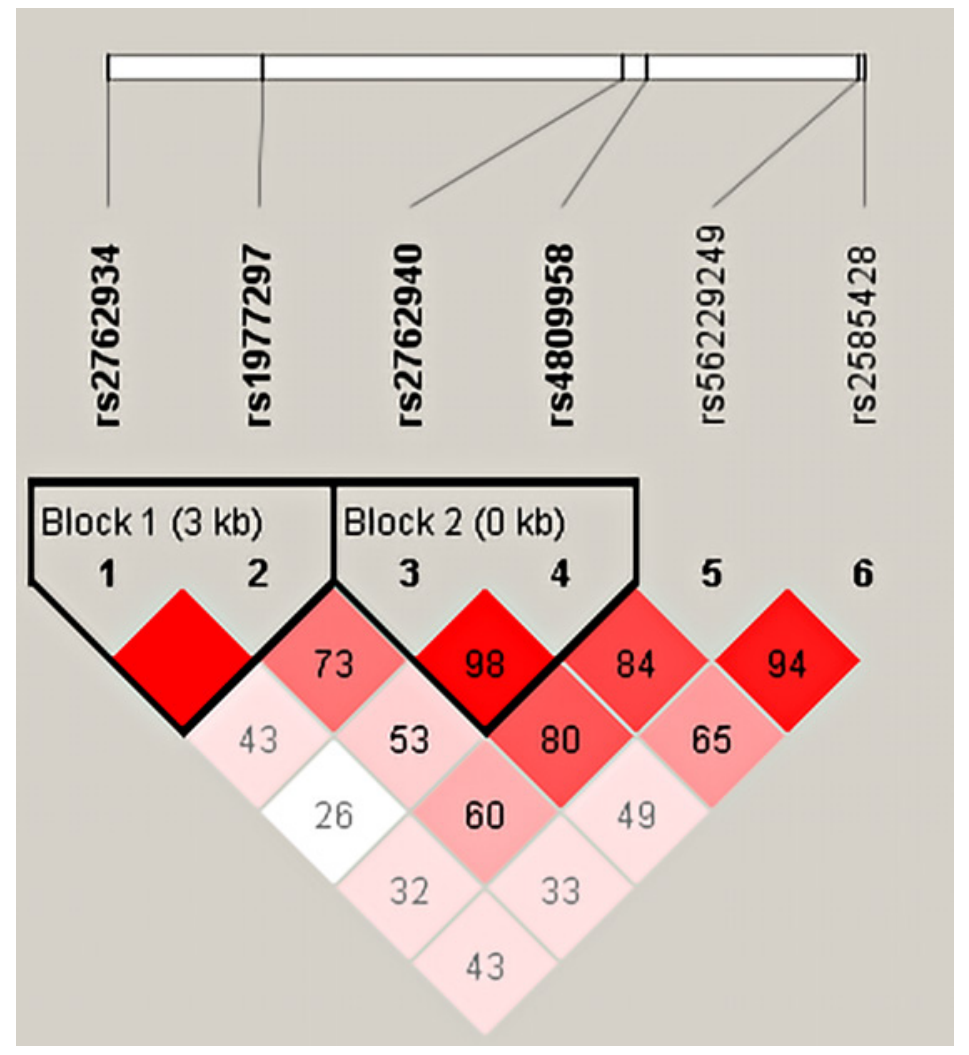

showed a significant difference between $C Y P 24 A 1$ rs2762934 and the low-density lipoprotein level $(p=0.036)$, and the level of UA was different in genotypes of CYP24A1 rs2762940 ( $p=$ $0.030)$.

\section{Discussion}

The present study investigated 6 SNPs of $C Y P 24 A 1$ and identified a significant association of 1 SNP (rs56229249) with the risk of hypertension. Specially, rs2762934 and rs1977297 significantly increased the hypertension risk in the subgroup of age. rs2762940 was associated with a higher hypertension risk in men and rs56229249 of CYP24A1 had a protective function on hypertension in women. We also found two blocks (block 1: rs2762934 and rs1977297; block 2: rs2762940 and rs4809958).

CYP24A1 is an important cytochrome in P450 enzymes, mainly expressed in the kidney. The CYP24A1 gene encodes the enzyme which plays a vital role in calcium homeostasis and the vitamin D endocrine system [20,21]. Vitamin D, especially free vitamin D, is closely related to pathological conditions [22]. Disruption of CYP24A1 in mice cause dysregulation of vitamin $\mathrm{D}$ metabolism [23]. The level of vitamin $\mathrm{D}$ has been reported as an important parameter for hypertension risk. To date, only a few studies have been conducted to assess associations between polymorphisms of the CYP24A1 gene and hypertension susceptibility. Xiaoman et al. [24] evaluated the association between rs48009957, rs6068816 of CYP24A1, and hypertension risk in the Chinese Han population. It was reported in the Women's Genome Health Study that rs2296241 of CYP24A1 showed associations with systolic BP, diastolic BP, mean arterial pressure, and pulse pressure [12]. Specifically, rs6013897 in the CYP24A1 gene region 
Table 5. Association between haplotypes of CYP24A1 and hypertension susceptibility

\begin{tabular}{llllll}
\hline SNP & Haplotype & $\begin{array}{l}\text { Frequency } \\
\text { in cases }\end{array}$ & $\begin{array}{l}\text { Frequency } \\
\text { in controls }\end{array}$ & $\begin{array}{l}\text { OR } \\
\text { (95\% CI) }\end{array}$ \\
\hline rs2762934|rs1977297 & GT & 0.332 & 0.305 & $1.12(0.93-1.36)$ & 0.239 \\
rs2762934|rs1977297 & AC & 0.110 & 0.105 & $1.07(0.80-1.43)$ & 0.630 \\
rs2762934|rs1977297 & GC & 0.442 & 0.410 & $1.14(0.95-1.36)$ & 0.158 \\
rs2762940|rs4809958 & AG & 0.346 & 0.354 & $0.96(0.80-1.15)$ & 0.648 \\
rs2762940|rs4809958 & CT & 0.303 & 0.293 & $1.03(0.85-1.25)$ & 0.759 \\
rs2762940|rs4809958 & AT & 0.649 & 0.650 & $0.98(0.81-1.17)$ & 0.804 \\
\hline
\end{tabular}

SNP, single nucleotide polymorphism; OR, odds ratio; CI, confidence interval.

had prominent significant associations with both systolic BP and diastolic BP [21]. In our study, a strong relationship between CYP24A1 polymorphisms and hypertension risk was found, which might predict that genetic variants of CYP24A1 significantly influence the susceptibility of hypertension.

Sex differences have been established in which men have a higher incidence of hypertension compared with women of the same age until the age of 60 years [25, 26]. Similarly, the increasing prevalence of hypertension with age has been demonstrated [27]. Hence, both can be regarded as genetic risks for hypertension. Then, when we further stratified by age and sex, we observed the strong relationships between genetic variants of CYP24A1 and hypertension risk in our study. Among them, rs2762934 increased hypertension risk in codominant and dominant models for the subjects less than 61 years old, whereas rs 1977297 was associated with higher hypertension risk in the subgroup of elderly individuals $(\geq 61$ years). This was consistent with previous studies and confirmed that hypertension risk is age dependent. In addition, rs2762940 is associated with a 1.42-fold risk of hypertension in men, while rs56229249 could be a protective factor for hypertension in women. The results may provide new possibilities for the individual treatment for hypertension. However, larger samples are required to validate the role of $C Y P 24 A 1$ polymorphisms on hypertension susceptibility.

There are also some limitations to our study. First, all samples were recruited from a hospital, which inevitably introduced selection bias. Second, we did not analyze the influence of other risk factors, such as lifestyle, family history, and other lesions, because of the limited information. Third, we could not analyze the association of CYP24A1 polymorphisms and more clinical factors because of the limited information we obtained from the study subjects. Hence, further experiments are needed to verify the associations between CYP24A1 polymorphisms and hypertension risk.

\section{Conclusion}

The current findings show that polymorphisms of CYP24A1 may represent important determinants of susceptibility to hypertension in the Chinese Han population and that their effects on disease risk are age and sex specific. Further functional studies and large, welldesigned studies are expected to provide more evidence on how genetic variants of CYP24A1 affect hypertension susceptibility. 


\section{Acknowledgements}

The authors gratefully acknowledge all of the participants in this study.

\section{Statement of Ethics}

Our study protocol was approved by the Affiliated Hospital of Inner Mongolia Medical University Ethics Committee, and written informed consent was obtained from all participants.

\section{Disclosure Statement}

The authors report no conflicts of interest in this work.

\section{Funding Sources}

No funding was obtained for this study.

\section{References}

1 Kearney PM, Whelton M, Reynolds K, Muntner P, Whelton PK, He J. Global burden of hypertension: analysis of worldwide data. Lancet. 2005 Jan;365(9455):217-23.

2 Zhao X, Yin X, Li X, Yan LL, Lam CT, Li S, et al. Using a low-sodium, high-potassium salt substitute to reduce blood pressure among Tibetans with high blood pressure: a patient-blinded randomized controlled trial. PLoS One. 2014 0ct;9(10):e110131.

3 Tomaselli GF. Introduction to a compendium on sudden cardiac death: epidemiology, mechanisms, and management. Circ Res. 2015 Jun;116(12):1883-6.

4 Lv D, Zhou D, Zhang Y, Zhang S, Zhu YM. Two obesity susceptibility loci in LYPLAL1 and ETV5 independently associated with childhood hypertension in Chinese population. Gene. 2017 Sep;627:284-9.

5 Franceschini N, Le TH. Genetics of hypertension: discoveries from the bench to human populations. Am J Physiol Renal Physiol. 2014 Jan;306(1):F1-11.

6 Waken RJ, de Las Fuentes L, Rao DC. A Review of the genetics of hypertension with a focus on gene-environment interactions. Curr Hypertens Rep. 2017 Mar;19(3):23.

7 Kyrou I, Chrousos GP, Tsigos C. Stress, visceral obesity, and metabolic complications. Ann NY Acad Sci. 2010; 1083:77-110.

8 Robinson-Cohen C, Lutsey PL, Kleber ME, Nielson CM, Mitchell BD, Bis JC, et al. Genetic variants associated with circulating parathyroid hormone. J Am Soc Nephrol. 2017 May;28(5):1553-65.

9 Saadi HF, Nicholls MG, Frampton CM, Benedict S, Yasin J. Serum 25-hydroxyvitamin D is not related to cardiac natriuretic peptide in nulliparous and lactating women. BMC Endocr Disord. 2009 Jan; 9(1):4-4.

10 Zehnder D, Bland R, Chana RS, Wheeler DC, Howie AJ, Williams MC, et al. Synthesis of 1,25-dihydroxyvitamin $\mathrm{D}(3)$ by human endothelial cells is regulated by inflammatory cytokines: a novel autocrine determinant of vascular cell adhesion. J Am Soc Nephrol. 2002 Mar;13(3):621-9.

11 Skaaby T. The relationship of vitamin D status to risk of cardiovascular disease and mortality. Dan Med J. 2015 Feb;62(2):B5008.

12 Wang L, Chu A, Buring JE, Ridker PM, Chasman DI, Sesso HD. Common genetic variations in the vitamin D pathway in relation to blood pressure. Am J Hypertens. 2014 Nov;27(11):1387-95.

13 Cheng YB, Guo QH, Zhang DY, Wang Y, Huang QF, Sheng CS, et al. Association of pulse wave velocity with single nucleotide polymorphisms related to parathyroid hormone. Blood Press. 2018 Aug;27(4):222-30.

14 Meusburger E, Mündlein A, Zitt E, Obermayer-Pietsch B, Kotzot D, Lhotta K. Medullary nephrocalcinosis in an adult patient with idiopathic infantile hypercalcaemia and a novel CYP24A1 mutation. Clin Kidney J. 2013 Apr; 6(2):211-5

15 Jing J, Pattaro C, Hoppmann A, Okada Y, Fox CS, Köttgen A; CKDGen Consortium. Combination of mouse models and genomewide association studies highlights novel genes associated with human kidney function. Kidney Int. 2016 Oct;90(4):764-73. 
16 Zhang W, Tao Q, Guo Z, Fu Y, Chen X, Shar PA, et al. Systems pharmacology dissection of the integrated treatment for cardiovascular and gastrointestinal disorders by traditional Chinese medicine. Sci Rep. 2016 Sep;6(1):32400.

17 Prosser DE, Kaufmann M, O'Leary B, Byford V, Jones G. Single A326G mutation converts human CYP24A1 from 25-OH-D3-24-hydroxylase into -23-hydroxylase, generating 1 $\alpha, 25-(\mathrm{OH}) 2 \mathrm{D} 3-26,23$-lactone. Proc Natl Acad Sci USA. 2007 Jul;104(31):12673-8.

18 He WQ, Qiao YN, Zhang CH, Peng YJ, Chen C, Wang P, et al. Role of myosin light chain kinase in regulation of basal blood pressure and maintenance of salt-induced hypertension. Am J Physiol Heart Circ Physiol. 2011 Aug;301(2):H584-91.

19 Kim JH, Shin HD, Park BL, Moon MK, Cho YM, Hwang YH, et al. SLC12A3 (solute carrier family 12 member [sodium/chloride] 3) polymorphisms are associated with end-stage renal disease in diabetic nephropathy. Diabetes. 2006 Mar;55(3):843-8.

20 Sakaki T, Kagawa N, Yamamoto K, Inouye K. Metabolism of vitamin D3 by cytochromes P450. Front Biosci. 2005;10(1):119.

21 Kunutsor SK, Burgess S, Munroe PB, Khan H. Vitamin D and high blood pressure: causal association or epiphenomenon? Eur J Epidemiol. 2014 Jan;29(1):1-14.

22 Tsuprykov O, Chen X, Hocher CF, Skoblo R, Lianghong Yin, Hocher B. Why should we measure free 25(OH) vitamin D? J Steroid Biochem Mol Biol. 2018 Jun;180:87-104.

23 Carpenter TO. CYP24A1 loss of function: clinical phenotype of monoallelic and biallelic mutations. J Steroid Biochem Mol Biol. 2017 Oct;173:337-40.

24 Xiaoman Y, Jian J, Ning Z, Haixia D, Yiyang Z. Associations of genetic polymorphisms of the vitamin D pathway with blood pressure in a Han Chinese population. Clin Exp Hypertens. 2019;41(5):460-5.

25 Yoon SS, Gu Q, Nwankwo T, Wright JD, Hong Y, Burt V. Trends in blood pressure among adults with hypertension: United States, 2003 to 2012. Hypertension. 2015 Jan;65(1):54-61.

26 Mozaffarian D, Benjamin EJ, Go AS, Arnett DK, Blaha MJ, Cushman M, et al.; Writing Group Members; American Heart Association Statistics Committee; Stroke Statistics Subcommittee. Heart disease and stroke statistics 2016 update: a report from the American Heart Association. Circulation. 2016 Jan;133(4):e38-360.

27 Musso CG, Alfie J. Resistant hypertension in the elderly-second line treatments: aldosterone antagonists, central alpha-agonist agents, alpha-adrenergic receptor blockers, direct vasodilators, and exogenous nitric oxide donors. Cardiovasc Hematol Agents Med Chem. 2015;12(3):170-3. 